\title{
Speed of simple arithmetic in bilinguals
}

\author{
LUCINDA McCLAIN and JUDY Y. SHIH HUANG \\ Marquette University, Milwaukee, Wisconsin 53233
}

\begin{abstract}
Four types of bilinguals solved simple addition problems that were auditorily presented in their preferred language, the language in which they first learned arithmetic, or in their nonpreferred language. Subjects responded in the language in which the problems were presented. Solution time averaged $.227 \mathrm{sec}$ faster in the preferred language and was an increasing linear function of the number of addition operations required. The intercepts of the preferred and nonpreferred language functions significantly differed, but the slopes did not. The preferred language advantage was attributed to faster encoding and/or response times in the preferred language. When bilinguals used only one of their languages in a given experimental session, encoding/response times in the two languages were equivalent and the preferred language advantage was eliminated.
\end{abstract}

Bilinguals have two languages in which to express themselves. Despite high proficiency in both languages, bilinguals frequently report that they prefer to do simple arithmetic in the language in which it was first learned. Published (Kolers, 1968) and unpublished anecdotal reports suggest that this preference is based on efficiency; bilinguals claim to be more efficient at performing arithmetic in one of their languages. However, there is little experimental evidence to support these claims.

Marsh and Maki (1976) sought experimental evidence for the greater efficiency of one language by measuring the time required for bilinguals to solve simple addition problems composed of single digits. Subjects were required to respond in their preferred language, the language in which they first learned arithmetic, or in their nonpreferred language. Subjects were faster in their preferred language. For both languages, reaction time (RT) was a linear increasing function of the number of addition operations (one, two, or three) required. The slopes of the two functions did not differ.

Citing Sternberg's (1969) methods of analyzing RT data to infer cognitive processes, Marsh and Maki (1976) attributed the 200 -msec intercept difference to translation time. If subjects solved all problems in their preferred language, additional time would be needed to translate the sum to the nonpreferred language on trials for which a nonpreferred language response was required. If subjects used an abstract representation to solve all prob. lems, and if subsequent translation to the nonpreferred language required more time than translation to the preferred language, extra time on nonpreferred language trials would also be needed. Marsh and Maki's data did not allow them to distinguish these alternative explanations.

Experiment 1 is based on a Marquette University master's thesis by Judy Y. Shih Huang, directed by Lucinda McClain. We thank Roberto Lopez for help with Experiment 2. Requests for reprints should be set to Lucinda McClain, Psychology Department, Marquette University, Milwaukee, Wisconsin 53233.
Marsh and Maki's (1976) visual presentation of the addition problems allowed subjects to encode and add numbers in their preferred language and simply translate the sum on trials for which a nonpreferred language response was required. We sought a method that would more strongly induce bilinguals to encode and calculate in their nonpreferred language. At the suggestion of several bilinguals, auditory presentation of problems was used to promote use of the preferred language on preferred language trials and the nonpreferred language on nonpreferred language trials.

The preferred language was redefined as the language in which the subject first learned arithmetic and the language in which the subject currently preferred to do arithmetic. The second criterion for the preferred language was added because several bilinguals, interviewed prior to the experiment, reported that the language they preferred to use for arithmetic was not the language in which arithmetic was first learned. These subjects were excluded from the study.

Although Marsh and Maki (1976) found no difference in the performance of highly and moderately bilingual individuals, degree of bilingualism was included as a variable in the present study. Moderately bilingual subjects were more fluent in their preferred than nonpreferred language; highly bilingual subjects were equally fluent in both languages. If simple arithmetic is more efficient when subjects use their preferred language, we expected language differences to be greater among moderately bilingual subjects.

\section{EXPERIMENT 1}

\section{Method}

Subjects. Two types of bilingual young adults participated. Ten reported Chinese (Mandarin) as their preferred language and English as their nonpreferred language (Chinese-English bilinguals). Ten reported English as their preferred language and Chinese as their nonpreferred language (English-Chinese bilinguals). The Chinese-English bilinguals were born and educated in 
the Republic of China and were students attending Milwaukee area universities. All Chinese-English bilinguals had lived in the U.S.A. at least 1 year. The English-Chinese bilinguals were born and/or received all their education in the U.S.A.; they were recruited from members of a local Chinese-American community group.

A 9-point self-rating scale was used to determine degree of bilingualism (Marsh \& Maki, 1976; Macnamara, 1967). A 1 indicated that the person knew only English; a 9 indicated only knowledge of Chinese; a 5 indicated equal fluency. Five high Chinese-English and five high English-Chinese bilinguals had scores between 4 and 6 . Five moderate English-Chinese bilinguals had scores of 2 or 3 , and five moderate Chinese-English bilinguals had scores of 7 or 8 . A total of 24 subjects were screened to obtain the 20 volunteers who participated.

Apparatus. The arithmetic problems were recorded on tape by a Chinese-English bilingual who was highly proficient in both languages and who spoke English with no detectable Chinese accent. The tape was presented over earphones. An electronic signal coincident with the end of each problem activated an Automated Data Systems clockcounter. A Lafayette voice-activated relay stopped the time. Accuracy and speed of responses to the nearest millisecond were manually recorded.

Materials. Addition problems required one $(p+q=)$, two $(p+q+r=)$, or three $(p+q+r+s=)$ operations. No digit was repeated within a problem (e.g., $p+p=$ or $p+q+p=$ ), as the repetition of digits has been shown to decrease RT (Parkman $\&$ Groen, 1971). The sums ranged from 10 to 17 .

It was essential that subjects refrain from adding numbers until each problem was completely presented. A pilot study indicated that subjects were adding as the numbers were presented and thus reducing the difficulty of two- and threeoperation problems. Two steps were taken to minimize subjects' tendency to calculate before the end of the presentation. Only the numbers themselves were presented (e.g., " $3,6,4$ "), and subtraction problems were intermixed with the addition problems. In this way, subjects were discouraged from performing calculations until they heard the instruction that followed each series of numbers. If the instruction was "add," subjects summed the series of numbers. If the instruction was "subtract," subjects took the last number in the series and subtracted it from the first number in the series. The RTs to subtraction problems were not analyzed.

The experimental session contained 120 problems, 72 addition and 48 subtraction problems. Subjects experienced four language shifts. The order was English, Chinese, English, Chinese for half the subjects and Chinese, English, Chinese, English for the remainder. The 30 problems in each shift were divided into three blocks of 10 . Each block contained two one-operation, two two-operation, and two three-operation addition problems, plus four subtraction problems, all presented in a random order. The same random order for number of operations and addition/ subtraction problems was repeated in each language shift; the problems themselves were different.

Procedure. After completing the self-rating scale, instructions taped in English were presented. English was used for instructing and debriefing all subjects. The recording emphasized that subjects should respond in the language in which the problem was presented and that they should respond as quickly and accurately as possible. Twelve practice trials containing problems in both languages were presented. A 10-sec interval occurred between all problems. If a subject did not respond in this time period, the trial was counted as an error. Responses in the wrong language as well as incorrect answers were considered errors.

\section{Results}

RT data. Figure 1 shows mean correct RTs in the preferred and nonpreferred languages for ChineseEnglish and English-Chinese bilinguals. The RTs were

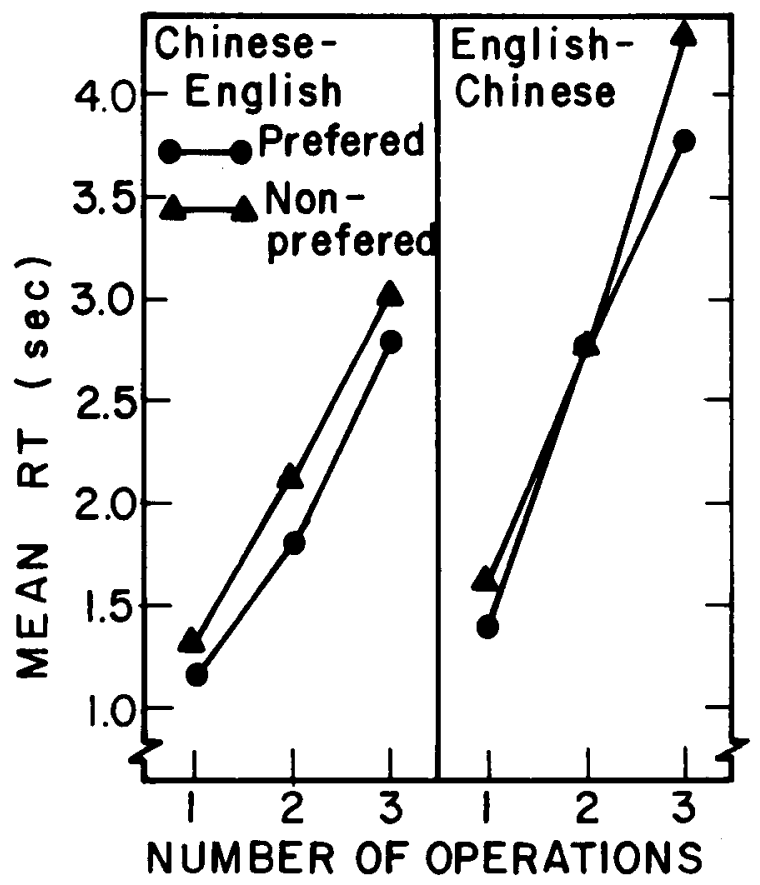

Figure 1. Mean reaction times of Chinese-English and EnglishChinese bilinguals as a function of number of addition operations and language in which problems were presented.

analyzed with a four-way analysis of variance: type of bilingual (Chinese-English and English-Chinese) by degree of bilingualism (high and moderate) by language (preferred and nonpreferred) by number of operations (one, two, and three). The Chinese-English bilinguals $(2.024 \mathrm{sec})$ were $.747 \mathrm{sec}$ faster than English-Chinese bilinguals $(2.771 \mathrm{sec})[\mathrm{F}(1,16)=8.16, \mathrm{MSe}=2.05 \mathrm{sec}]$, which is significant beyond the .05 level, the level of significance used in all subsequent tests. Subjects were $.225 \mathrm{sec}$ faster in their preferred language $(2.285 \mathrm{sec})$ than in their nonpreferred language $(2.510 \mathrm{sec})[\mathrm{F}(1,16)$ $=5.76, \mathrm{MSe}=.26]$. RT increased significantly with number of operations $[\mathrm{F}(2,32)=166.99, \mathrm{MSe}=.27]$, suggesting that the procedural steps taken to reduce the probability that bilinguals would begin addition operations before the problems were completely presented were at least partially effective. There was a significant Type of Bilingual by Number of Operations interaction $[\mathrm{F}(2,32)=7.54, \mathrm{MSe}=.27]$, produced by the fact that English-Chinese bilinguals were significantly slower than Chinese-English bilinguals on twooperation and three-operation problems, but not on oneoperation problems. Degree of bilingualism and all other interactions were not significant.

To facilitate comparison with the previous research (Marsh \& Maki, 1976), linear equations for the preferred language $(\overline{\mathrm{RT}}=.230+1.025 \mathrm{x})$ and nonpreferred language $(\overline{\mathrm{RT}}=.350+1.082 \mathrm{x}) \mathrm{RT}$ functions were computed. Equivalent slopes $[F(1,19)<1]$ for the preferred and nonpreferred language functions were expected in light of the nonsignificant Language by Number of 
Operations interaction. The preferred language advantage was reflected as a significant difference in the intercepts of the RT functions $[F(1,19)=4.50$, MSe $=$ $.03]$.

Error data. The error rate was $2.2 \%$. Of these, $84 \%$ were incorrect answers, $14 \%$ were omissions, and $2 \%$ were wrong language responses. Problems involving one or two operations produced fewer errors than problems involving three operations. Otherwise, errors were evenly distributed over the experimental conditions.

\section{EXPERIMENT 2}

One purpose of Experiment 2 was to replicate Experiment 1 using Spanish-English and English-Spanish bilinguals, the type of bilinguals used by Marsh and Maki (1976). A second purpose was to compare bilinguals and monolinguals. Marsh and Maki reported that English speakers were faster solving visually presented addition problems than English-Spanish bilinguals performing in English, their preferred language. To determine if a similar difference would occur with auditory presentation, we included a monolingual comparison group in Experiment 2.

Experiment 2 also included bilingual subjects who solved problems in only one of their languages during a given experimental session. The purpose of this group was to ascertain whether the slower performance of bilinguals relative to monolinguals (Marsh \& Maki, 1976) was produced by bilinguals' having to switch between languages. If switching time were responsible, we expected the performance of bilinguals who used only one language in a session to be faster than the performance of bilinguals who used two languages. If switching time were completely responsible for the bilingual-monolingual performance difference, we expected this difference to disappear when bilinguals used only one language in a session.

\section{Method}

Subjects. Forty bilingual adults participated; 20 reported Spanish as their preferred language (Spanish-English bilinguals) and 20 reported English as their preferred language (EnglishSpanish bilinguals). The Spanish-English bilinguals, born and educated in eight Spanish-speaking countries, were attending Milwaukee area universities. The English-Spanish bilinguals, born and/or educated in the U.S.A., were also university students. Most of these subjects came from Spanish-speaking homes. About half the bilinguals in each group were undergraduates; the remainder were graduate students. Most were members of an association for Hispanic students.

Degree of bilingualism was not included as a variable. Subjects had scores between 3 and 7 on a 9-point self-rating scale similar to that used in Experiment 1. Median scores were 4 and 6 in the English-Spanish and Spanish-English groups, respectively.

Twenty English monolingual university undergraduates also volunteered. All students had scores of 1 on the self-rating scale, and none was studying Spanish. Eight of these students had studied another foreign language in college, but none considered himself/herself even minimally fluent in that language.
Materials and Apparatus. The apparatus and materials from Experiment 1 were used, except that problems presented in Spanish were substituted for those presented in Chinese. The tape was recorded by a Spanish-English bilingual who was highly proficient in both languages and who spoke English with no detectable Spanish accent. In addition, a tape containing all 120 problems presented in English was recorded by the same speaker.

Procedure. The experimental procedure was identical to that used in Experiment 1 for the 10 Spanish-English and 10 EnglishSpanish bilinguals, who worked in both languages in one session. Half received the Spanish, English, Spanish, English presentation order, and half received the opposite order.

The 20 bilinguals who participated in two sessions worked the same total number of problems. Five Spanish-English and five English-Spanish bilinguals received the 60 Spanish problems the 1 st day and the 60 English problems the 2nd day. The remaining bilingual subjects received the opposite presentation order. Appointments for the two sessions were $17-40 \mathrm{~h}$ apart. Assignment of bilinguals to one or two sessions was randomly determined.

Ten monolinguals worked 120 English problems in one session. Ten worked 60 English problems in one session. Assignment to these groups was randomly determined.

Prior to the experiment, the bilinguals participated in a simple number naming task. The numbers 1-17 were auditorily presented and subjects repeated each number in the language in which it had been presented. Number naming times were recorded to the nearest millisecond. Bilinguals who were to participate in one session in Experiment 2 experienced four language shifts in number naming. The order was Spanish, English, Spanish, English for half the subjects and English, Spanish, English, Spanish for the remainder. Each number was presented twice in each shift, for a total of 134 trials. The order of the numbers within each shift was randomly determined independently.

Bilinguals who were to participate in two sessions in Experiment 2 also participated in two number naming sessions. Half the subjects received the 68 numbers presented in Spanish at the first session and the 68 numbers presented in English at the second session. The remainder of these subjects received numbers in the two languages in the opposite order. The two sessions occurred $24-45 \mathrm{~h}$ apart. The number naming task took place 1 week prior to the arithmetic session(s).

\section{Results}

Bilingual RT data. Correct RTs were analyzed with a four-way analysis of variance: sessions by type of bilingual by language by number of operations. The means from this analysis are shown in Figure 2. Bilinguals who performed in both languages at one session $(2.458 \mathrm{sec})$ were $.481 \mathrm{sec}$ slower than bilinguals who participated in two sessions $(1.977 \mathrm{sec})[\mathrm{F}(1,36)=8.10$, MSe $=1.71 \mathrm{sec}] . \quad$ The Spanish-English bilinguals $(1.824 \mathrm{sec})$ were $.786 \mathrm{sec}$ faster than the English-Spanish bilinguals $(2.610 \mathrm{sec}) \quad[\mathrm{F}(1,36)=21.61, \mathrm{MSe}=1.71]$. There was no main effect of language, but the Sessions by Language interaction was significant $[F(1,36)=4.58$, $\mathrm{MSe}=.22]$. Performance in the preferred $(1.991 \mathrm{sec})$ and nonpreferred $(1.962 \mathrm{sec})$ languages of bilinguals who used only one language per session did not significantly differ. When both languages were used in one session, a significant difference of $.229 \mathrm{sec}$ between preferred $(2.343 \mathrm{sec})$ and nonpreferred $(2.572 \mathrm{sec})$ languages was found. 


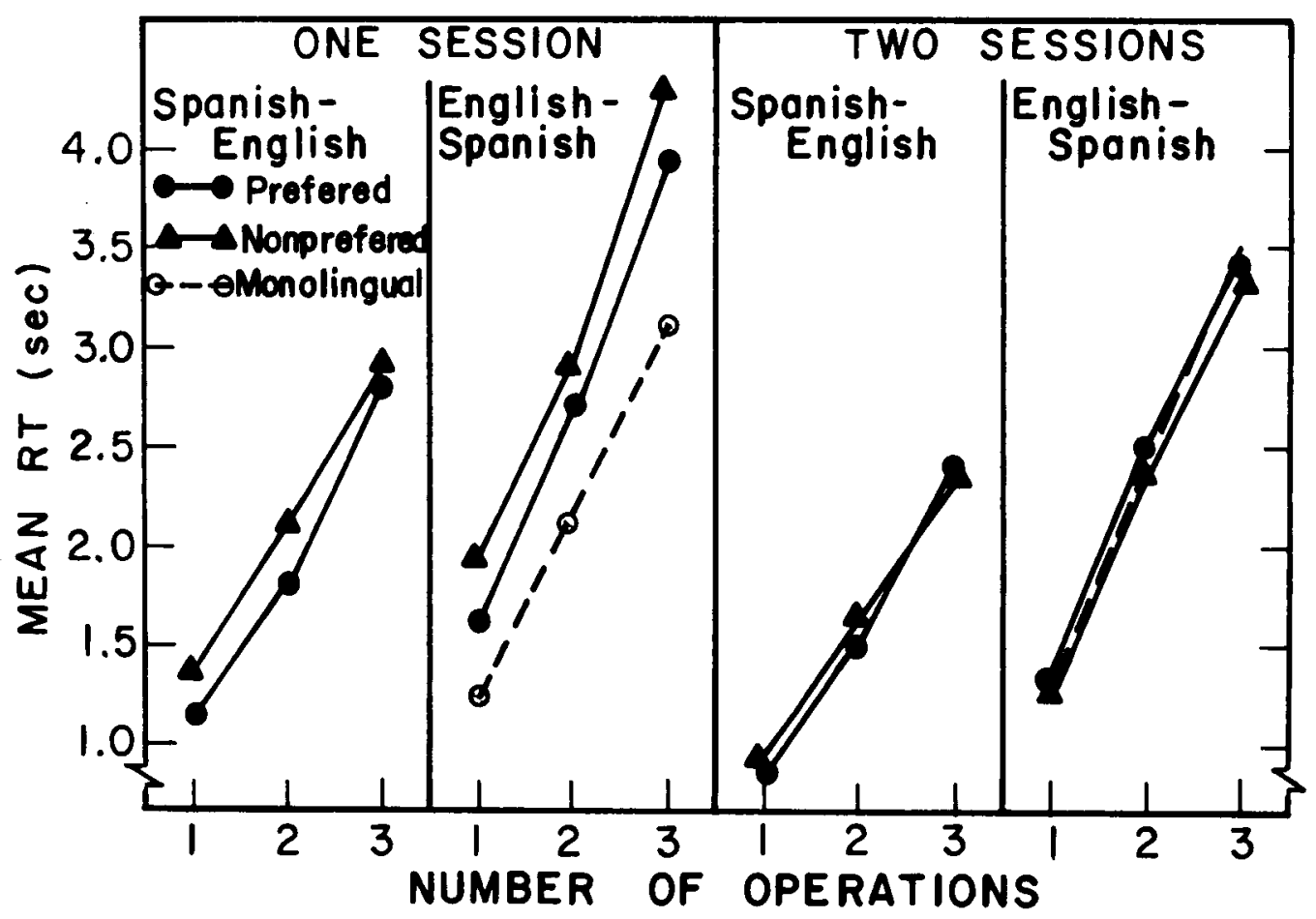

Figure 2. Mean reaction times of Spanish-English and English-Spanish bilinguals as a function of number of sessions, number of addition operations, and language in which problems were presented. Reaction times for English monolingual comparison groups are also shown.

RT increased significantly with number of operations $[\mathrm{F}(2,72)=282.29, \mathrm{MSe}=.26]$, and number of operations interacted with type of bilingual $[F(2,72)$ $=8.23, \mathrm{MSe}=.26 \mathrm{]}$. The difference between SpanishEnglish and English-Spanish bilinguals grew larger as the number of operations increased. All other interactions were not significant.

Linear equations for the RT functions in both languages were computed. For bilinguals who participated in one session, the preferred language function was $\overline{\mathrm{RT}}=.337+1.003 \mathrm{x}$ and the nonpreferred language function was $\overline{\mathrm{RT}}=.604+.984 \mathrm{x}$. The intercepts of these functions were significantly different $[F(1,19)=6.05$, $\mathrm{MSe}=.12]$, but the slopes were not $[\mathrm{F}(1,19)<1]$. For bilinguals who participated in two sessions, the preferred language function was $\overline{\mathrm{RT}}=.141+.925 \mathrm{x}$ and the nonpreferred language function was $\overline{\mathrm{RT}}=.184+.889 \mathrm{x}$. Neither the slopes nor the intercepts of these two functions differed [both $\mathrm{Fs}(1,19)<1]$.

Comparison with monolinguals. The preferred language performance of English-Spanish bilinguals who participated in one session was compared with the performance of the 10 English monolinguals who solved 120 problems in one session using a type of speaker by number of operations analysis of variance. Only 36 of the 72 addition problems performed by the monolinguals were anlayzed, in order that the specific English problems solved by the two groups would be identical. Monolinguals $(2.116 \mathrm{sec})$ were $.625 \mathrm{sec}$ faster than
English-Spanish bilinguals performing in their preferred language $(2.741 \mathrm{sec})[\mathrm{F}(1,18)=6.91, \mathrm{MSe}=.72]$. As expected, the main effect of number of operations was significant $[F(2,36)=158.55, \mathrm{MSe}=.14]$; the interaction was not significant.

The preferred language performance of EnglishSpanish bilinguals who participated in two sessions was compared with the performance of the 10 English monolinguals who solved 60 problems in one session using a type of speaker by number of operations analysis of variance. Only the main effect of number of operations was significant $[F(2,36)=149.68, M S e=.16]$. The performance of monolinguals $(2.414 \mathrm{sec})$ and bilinguals using their preferred language $(2.397 \mathrm{sec})$ did not significantly differ.

Error data. The error rate was $3.1 \%$. Of these, $91 \%$ were incorrect answers, 6\% were omissions, and 3\% were wrong language responses. Subjects in all groups made fewer errors on one- and two-operation than on three-operation problems. Otherwise, errors were evenly distributed across conditions.

Number naming. The correct number naming times were analyzed with a sessions by type of bilingual by language analysis of variance. Subjects who participated in two sessions had naming times $(.216 \mathrm{sec})$ that were $.184 \mathrm{sec}$ faster than those who participated in one session $(.400 \mathrm{sec})[\mathrm{F}(1,36)=16.89, \mathrm{MSe}=.04]$. Subjects were $.070 \mathrm{sec}$ faster naming numbers presented in their preferred language $(.273 \mathrm{sec})$ than in their nonpreferred 
language $(.343 \mathrm{sec}) \quad[\mathrm{F}(1,36)=19.38, \quad \mathrm{MSe}=.005]$. However, there was a significant Sessions by Language interaction $[F(1,36)=11.12, \mathrm{MSe}=.005]$. Bilinguals who participated in one session exhibited a significant .122 -sec preferred language advantage in number naming (.339 vs. $.461 \mathrm{sec}$ ), but the naming times of bilinguals who participated in two sessions did not significantly differ $(.208$ vs. $.225 \mathrm{sec})$. The main effect of type of bilingual and all other interactions were not significant. The error rate was extremely low (.001\%).

\section{DISCUSSION}

Our auditory presentation method was designed to induce bilinguals to perform simple arithmetic problems in the language in which the problems were presented and was intended to correct an interpretative difficulty that arose as a consequence of the visual presentation method used by Marsh and Maki (1976). Improving the methodology, we still found a preferred language advantage when bilinguals used both languages in one experimental session; that is, subjects solved addition problems presented in their preferred language faster than problems presented in their nonpreferred language. As in the previous study (Marsh \& Maki, 1976), the intercepts of the preferred language RT functions were lower than the intercepts of the nonpreferred language RT functions, and the slopes of the two RT functions did not differ. The intercept of an RT function is assumed to reflect encoding and response times, and the slope presumably reflects the duration of other cognitive operations (Sternberg, 1969). The pattern of results we obtained suggests that the preferred language advantage was produced by encoding and/or response processes that were faster in the preferred language. Once the digits were encoded, computations proceeded at the same rate in both languages.

The suggestion that the preferred language advantage was due to faster encoding and/or response times received support from the results of the number naming task in Experiment 2. Number naming required only that subjects encode a number and respond with its name, and RTs in this task were faster in the preferred language than in the nonpreferred language. The preferred language advantage in number naming was $.070 \mathrm{sec}$, whereas the intercept difference in Experiment 2 was $.267 \mathrm{sec}$. Because number naming and addition were different tasks, the difference in these values cannot be unequivocally interpreted.

Translating between languages is an encoding/response process and would be expected to affect the intercept of an RT function, as Marsh and Maki (1976) noted. Their visual presentation method allowed subjects to encode the digits in the preferred language on all trials and to simply translate the sum to the nonpreferred language on trials for which a nonpreferred language response was required. They reported that this explanation coincided with the subjective reports of many of their bilinguals. In the present study, it is possible that our intercept difference also reflects translation time. Subjects could have translated digits presented in the nonpreferred language to the preferred language before beginning calculations and then translated the answer back to the nonpreferred language before responding. This strategy would have required two translation steps, and none of our subjects reported using this method in postexperimental interviews.

An unexpected finding occurred in both Experiments 1 and 2. Bilinguals with English as their nonpreferred language (Chinese-English and Spanish-English bilinguals) were faster at solving addition problems than bilinguals with English as their preferred language. Given the correlational nature of this relationship, we cannot offer an explanation. The greater emphasis on drill in early mathematics training frequently found in foreign countries or the special characteristics of students who study abroad provides appealing speculations.

As expected, and consistent with previous results (Marsh \& Maki, 1976), Experiment 2 showed that English monolinguals performed faster than EnglishSpanish bilinguals using English, their preferred language. The slower performance of the bilinguals can be attributed to their having to switch languages within an experimental session. When bilinguals were required to use only one of their languages in a given session, their performance was significantly faster and did not differ from the monolingual comparison group. The deleterious effects of language switching have been previously reported (Macnamara, Krauthammer, \& Bolgar, 1968), using a visually presented digit naming task and more frequent, unpredictable switches. Experiment 2 showed that as few as three planned switches in an experimental session can slow the speed of bilingual arithmetic.

Our suggestion that the preferred language advantage was produced by faster encoding and/or response times when subjects used both languages in one session received additional support from the number naming data of bilinguals who participated in two experimental sessions. When bilinguals used only one of their languages per session, number naming times in the two languages did not differ and the preferred language advantage in arithmetic was eliminated. Apparently, encoding and response times are influenced only when subjects are prepared to work in both languages over a short period and are required to switch between languages.

Our study demonstrated that the preferred language advantage previously reported (Marsh \& Maki, 1976) does not depend on visual presentation of the problems and that the preferred language advantage can be eliminated by requiring bilinguals to use only one of their languages in an experimental session. Future research should be directed toward determining whether encoding or response times are primarily responsible for the preferred language advantage when bilinguals work actively in both of their languages. 


\section{REFERENCES}

Kolers, P. A. Bilingualism and information processing. Scientific American, 1968, 218, 78-86.

Marsh, L. G., \& MAKI, R. H. Efficiency of arithmetic operations in bilinguals as a function of language. Memory \& Cognition, 1976, 4, 459-464.

Macnamara, J. The bilinguals' linguistic performance-A psychological overview. Journal of Social Issues, 1967, 23, 58-77.

Macnamara, J., Krauthammer, M., \& Bolgar, M. Language switching in bilinguals as a function of stimulus and response uncertainty. Journal of Experimental Psychology, 1968, 78, 208-215.

Parkman, M., \& Groen, J. Temporal aspects of simple addition and comparison. Journal of Experimental Psychology, 1971, 89, 335-342.

Sternberg, S. Memory-scanning: Mental processes revealed by reaction-time experiments. American Scientist, 1969, 57, 421457.

(Received for publication July 28, 1981; revision accepted July 21,1982 .) 